\title{
Intergenerational genomic DNA methylation patterns in mouse hybrid strains
}

\author{
Luz D Orozco ${ }^{1}$, Liudmilla Rubbi ${ }^{1}$, Lisa J Martin ${ }^{2}$, Fang Fang ${ }^{3}$, Farhad Hormozdiari ${ }^{4}$, Nam Che ${ }^{2}$, Andrew D Smith ${ }^{3}$, \\ Aldons J Lusis ${ }^{2}$ and Matteo Pellegrini ${ }^{1 *}$
}

\begin{abstract}
Background: DNA methylation is a contributing factor to both rare and common human diseases, and plays a major role in development and gene silencing. While the variation of DNA methylation among individuals has been partially characterized, the degree to which methylation patterns are preserved across generations is still poorly understood. To determine the extent of methylation differences between two generations of mice we examined DNA methylation patterns in the livers of eight parental and F1 mice from C57BL/6J and DBA/2J mouse strains using bisulfite sequencing.

Results: We find a large proportion of reproducible methylation differences between C57BL/6J and DBA/2J chromosomes in CpGs, which are highly heritable between parent and F1 mice. We also find sex differences in methylation levels in 396 genes, and 11\% of these are differentially expressed between females and males. Using a recently developed approach to identify allelically methylated regions independently of genotypic differences, we identify 112 novel putative imprinted genes and microRNAs, and validate imprinting at the RNA level in 10 of these genes.

Conclusions: The majority of DNA methylation differences among individuals are associated with genetic differences, and a much smaller proportion of these epigenetic differences are due to sex, imprinting or stochastic intergenerational effects. Epigenetic differences can be a determining factor in heritable traits and should be considered in association studies for molecular and clinical traits, as we observed that methylation differences in the mouse model are highly heritable and can have functional consequences on molecular traits such as gene expression.
\end{abstract}

\section{Background}

Methylation of DNA cytosine bases is essential for mammalian development and plays an important role in $\mathrm{X}$-chromosome inactivation, imprinting, regulation of gene expression, silencing of repetitive elements and differentiation. Aberrant DNA methylation in early development is lethal, and in adult humans it is associated with both rare and complex diseases, such as Prader-Willi syndrome, Angelman syndrome, cancer, aging [1] and rheumatoid arthritis [2]. In mammals, DNA methylation occurs primarily on cytosines of CG dinucleotides, although high methylation in the $\mathrm{CHG}$ and $\mathrm{CHH}$ sequence contexts (where $\mathrm{H}$ is any base other than $\mathrm{G}$ ) has been observed in stem cells [3] and in adult mouse frontal cortex [4]. The genome contains CG-rich regions known as CpG islands

\footnotetext{
* Correspondence: matteop@mcdb.ucla.edu

'Department of Molecular, Cell and Developmental Biology, University of California Los Angeles, Los Angeles, CA 90095, USA

Full list of author information is available at the end of the article
}

that are predominantly unmethylated and associated with transcriptional start sites. In the remainder of the genome, CpGs are generally sparse, and these CpGs tend to be highly methylated [5-7]. While the regulation of DNA methylation by numerous enzymes has been well studied, the transgenerational inheritance of DNA methylation patterns and their relationship with genetic variation is only beginning to be elucidated.

During gametogenesis and embryogenesis, epigenetic reprogramming of the cell involves global changes in epigenetic marks, and DNA methylation patterns are erased and re-established in the progeny. A small fraction of genetic loci can escape epigenetic reprogramming and thus can be stably transmitted to the next generation $[8,9]$, and epigenetic inheritance patterns can be influenced by both environmental [10] and genetic variation [11]. Nonetheless, to date few studies have examined transgenerational inheritance of DNA methylation patterns using genome-wide approaches, and the degree to which these

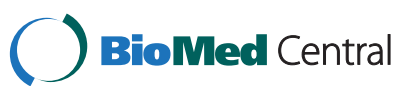


patterns are faithfully reproduced across generations remains to be determined. To address these questions, we examined the influence of genetics, sex and parental origin effects on intergenerational DNA methylation profiles in the mouse liver. We constructed reciprocal crosses of the widely used laboratory mouse strains C57BL/6J and DBA/2J, and examined global DNA methylation patterns in the parents, their F1 progeny and in both females and males using reduced representation bisulfite sequencing.

\section{Results}

\section{Reduced representation bisulfite sequencing data}

We made reciprocal crosses of the two genetically distinct mouse inbred strains C57BL/6J (B6) and DBA/2J (DBA), and constructed reduced representation bisulfite sequencing (RRBS) libraries from liver genomic DNA of female and male B6 and DBA parents, from BXD F1 mice where the female parent is $\mathrm{B} 6$ and male parent is DBA, and from DXB F1 mice where the female parent is DBA and male parent is B6 (Figure 1A). We used the Illumina HiSeq to sequence the RRBS libraries and obtained, on average, 96.2 million reads (Figure S1A in Additional file 1). On average, we uniquely aligned 21.9 million reads to the mouse genome using BS Seeker [12], which corresponded to $25.6 \%$ mappability (Figure S1B in Additional file 1) and $60 \times$ average coverage (Figure S1C in Additional file 1). To estimate the sequencing error rate in our data, we compared base calls in data from B6 and DBA parents to published genotypes at known polymorphic SNPs, using publicly available data from the Wellcome Trust Sanger sequencing mouse genomes project [13]. We found that approximately $99.02 \%$ of the polymorphic reads correctly match the expected genotype in B6 (99.2\%) and DBA (99.1\%) mice, and approximately $0.98 \%$ of the reads did not match the expected genomic sequence. Hence, we conclude that the sequencing error rate in our RRBS data was $<1 \%$ (Figure S1D in Additional file 1). Furthermore, we examined global DNA methylation levels and found no significant differences among the different samples, for which the overall cytosine methylation rate was $8.05 \% \pm 1.72 \%$, the CG methylation rate was $48.39 \pm 7.71 \%$, the CHG methylation rate was $0.71 \% \pm 0.26 \%$ and the $\mathrm{CHH}$ methylation rate was $0.75 \% \pm 0.26 \%$ (Figure S1E,F in Additional file 1). Global methylation levels were consistent with previous studies in mammalian somatic cells [6].

\section{Strain-specific methylation}

To determine if individual sites in the genome were differentially methylated between the strains, we first compared individual sites between the parental B6 and DBA female mice, and between parental B6 and DBA male mice, using a binomial test [14]. We restricted our analysis to sites covered by at least 10 reads, and categorized a site as differentially methylated if the methylation level of each strain was outside of the $95 \%$ confidence interval of methylation of each other, and the absolute difference in percentage methylation between the two samples was greater than $50 \%$, with false discovery rate (FDR) $<1 \%$. Using these criteria, we found 6,247 cytosine sites differentially methylated between B6 and DBA female parents, and 8,480 cytosines differentially methylated between B6 and DBA male parents. This corresponded to an overall allele-specific methylation rate of $0.10 \%$ (approximately $1 / 1,000$ cytosines) in female parents, and $0.12 \%$ in males parents $(1 / 850$ cytosines). This rate was higher for CG methylation $(0.56 \%$ and $0.52 \%$ in females and males) than for either CHG $(0.03 \%$ and $0.06 \%)$ or $\mathrm{CHH}(0.04 \%$ and $0.03 \%)$ methylation. The genome-wide distribution of these sites is shown in Figure 1B for females and in Figure S2A in Additional file 2 for males. Although most of the cytosines in the mouse genome are non-CG, we observed that the rate of differential methylation was approximately five times higher in CGs $(0.56 \%)$ relative to all cytosines $(0.10 \%)$. Indeed, $75 \%$ of differentially methylated cytosines in the female parents were CGs, and $71 \%$ were in the male parents (Figure S2B,C in Additional file 2). We also observed that, of the sites that were represented in both female and male parent datasets, there was a significant overlap of 2,865 cytosines (hypergeometric $P<1 \times 10^{-16}$ ), and the remaining were unique to either females or males (Figure S2D in Additional file 2).

To determine the reproducibility and the degree of variation in our bisulfite sequencing data, we generated bisulfite sequencing libraries of technical replicates (different libraries from the same mouse genomic DNA sample) and biological replicates (different mice from the same strain), and looked for the presence of differentially methylated cytosines using the binomial test to compare methylation levels between pairs of samples, as described above. We found, on average, 383 differentially methylated cytosines when comparing technical replicates, and 524 differentially methylated cytosines between biological replicates (Figure 2A). In contrast, on average, we identified 7,364 differentially methylated cytosines when comparing samples from different mouse strains, which was 20 -fold and 14-fold higher than the number of differentially methylated sites we identified in technical and biological replicates, respectively. Furthermore, we would expect to find a large false positive rate if variation in methylation levels between technical or biological samples was similar to the variation among different mouse strains. Therefore, to examine the degree of variation in methylation levels derived from bisulfite sequencing data, we compared the distribution of the variance in methylation levels between biological replicates (intra-strain variance) to the variance in methylation between different mouse strains (inter-strain variance) using the Kolmogorov-Smirnov (KS) test. We found that the variance in methylation levels 
A

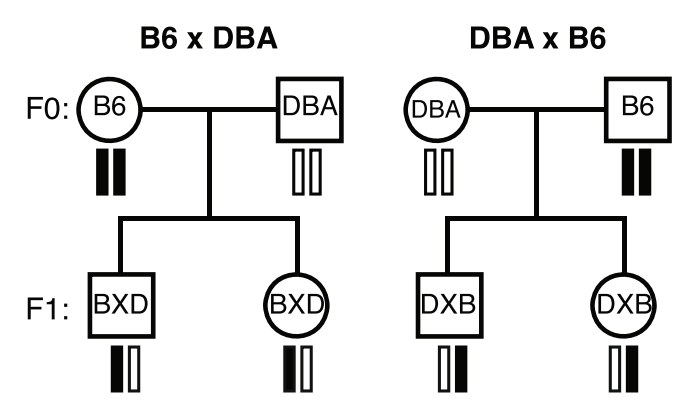

C

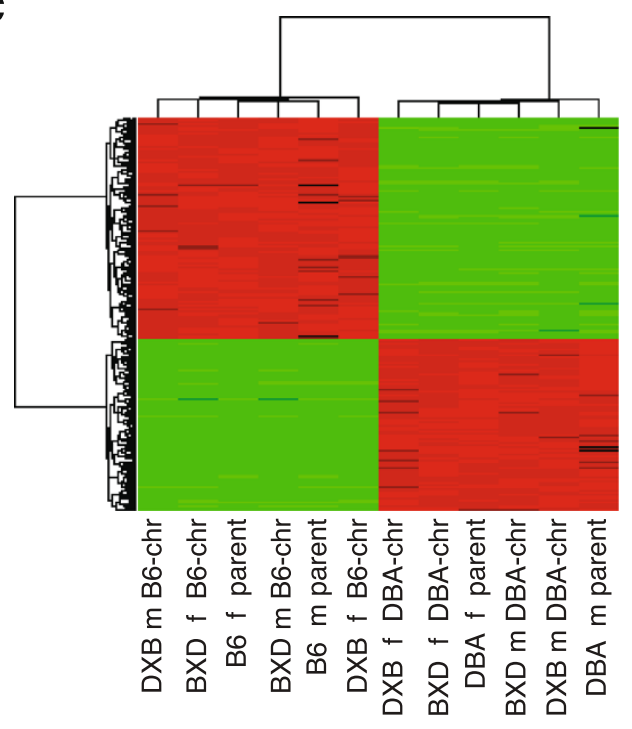

B

Strain-specific methylation in B6 and DBA parents

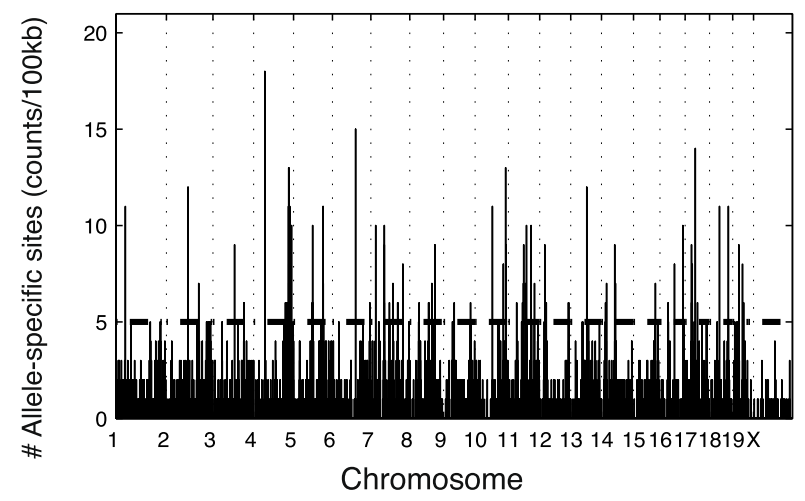

D

Allele-specific methylation in all samples

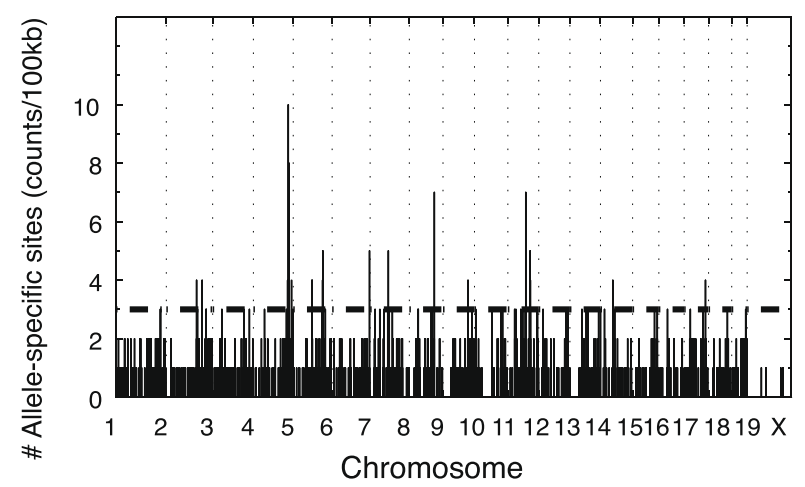

E

\begin{tabular}{|c|c|c|c|c|c|}
\hline \multicolumn{6}{|l|}{ Sequence $\Rightarrow$} \\
\hline Refseq genes & Hspg2 & & & & \\
\hline B6 parent $F$ - & $\mid \begin{array}{l}100 \% \\
0 \%\end{array}$ & & & & \\
\hline B6 parent M - & & & & & \\
\hline B6chr F1 BXD F - & $\mid \begin{array}{l}100 \% \\
0 \% \\
100 \%\end{array}$ & & & & \\
\hline $\begin{array}{l}\text { B6chr F1 BXD M - } \\
\text { B6chr F1 DXB F - }\end{array}$ & $\begin{array}{l}0 \% \% \\
100 \%\end{array}$ & & & & \\
\hline $\begin{array}{l}\text { B6chr F1 DXB F - } \\
\text { B6chr F1 DXB M - }\end{array}$ & 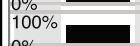 & & & & \\
\hline DBAchr F1 BXD F - & $100 \%$ & 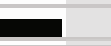 & & & \\
\hline DBAchr F1 BXD M & $100 \%$ & 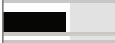 & & & \\
\hline DBAchr F1 DXB F - & $100 \%$ & & & & \\
\hline DBAchr F1 DXB M & $100 \%$ & & & & \\
\hline DBA parent $F$ - & $\begin{array}{l}100 \% \\
0 \% \% \\
110 \%\end{array}$ & $\boldsymbol{E}$ & & & \\
\hline DBA parent M - & $\mid 00 \%$ & & & & \\
\hline
\end{tabular}

Figure 1 (See legend on next page.) 
(See figure on previous page.)

Figure 1 Allele-specific methylation. (A) Pedigree illustrating design of the mouse cross. Circles denote females and squares males. Black bars represent chromosomes derived from the C57BL/6J parents (B6) and white bars are chromosomes derived from the DBA/2J parents (DBA). (B) Allele-specific methylation sites between B6 and DBA female parents. The number of cytosines differentially methylated in each 100-kb bin is shown on the $Y$-axis and the genomic position of the bin is on the X-axis. All sites plotted are significant, and the horizontal dashed line represents the significance threshold for the 100-kb bin. (C) Hierarchical clustering of mice (columns) and allele-specific methylation sites (rows) for $\mathrm{CG}, \mathrm{CHG}$ and $\mathrm{CHH}$ differentially methylated cytosines. Red indicates increased methylation and green indicates decreased methylation relative to other samples. $f$, female; $m$, male. (D) Allele-specific methylation sites identified using all eight mice. The number of $C$ residues in each bin is on the $\mathrm{Y}$-axis and the genomic position is on the X-axis. All sites plotted are significant, and the horizontal dashed line represents the significance threshold for each bin. (E) Methylation levels across all samples, showing allele-specific methylation of the gene Hspg2. The height of the bar represents percentage methylation from 0 to 100\%. Gray represents missing data. The minus sign at the end of each sample denotes data from the minus strand.

between strains was 9-fold higher than the variance in methylation between biological replicates (KS test $P<1 \times$ $10^{-16}$; Figure $2 \mathrm{~B}$ ) for all cytosines, and 2.4-fold higher for CpG methylation levels (KS test $P<1 \times 10^{-16}$; Figure $2 \mathrm{C}$ ).

\section{Allele-specific methylation}

We next examined methylation levels across all samples in order to identify allele-specific methylation events that persist across inbred parents and hybrid progeny. To analyze data in the hybrid mice we first used the bisulfite sequences to identify SNPs between the two strains, and found 24,132 SNPs between B6 and DBA represented in our sequenced fragments. We used these SNPs to determine whether individual reads in the hybrid mice were derived from the $\mathrm{B} 6$ or DBA chromosomes, and found 127,893 cytosines in polymorphic reads tagged by SNPs. Next, we examined allele-specific methylation of reads derived from the $\mathrm{B} 6$ chromosomes and compared them to reads from the DBA chromosomes using a $t$-test. We selected sites where $P<0.05$ and the difference in methylation was greater than $50 \%$ (FDR $<0.05 \%$ ). Here we found 2,091 differentially methylated sites, which corresponded to an allele-specific methylation rate of $5.3 \%$ for CG, $0.77 \%$ for $\mathrm{CHG}$ and $0.88 \%$ for $\mathrm{CHH}$ contexts. A clustering heatmap for differentially methylated cytosines of all contexts is shown in Figure $1 \mathrm{C}$ and the genome-wide distribution is shown in Figure 1D. We also observed that reads from all B6 chromosomes cluster together, separately from DBA chromosomes, even when using data from all polymorphic sites present in all samples (Figure S3 in Additional file 3). As an example, a cluster of CpGs in the gene encoding heparan sulfate proteoglycan 2 (Hpsg2) showed large differences in methylation between B6 and DBA chromosomes. Methylation levels across all samples are shown in Figure $1 \mathrm{E}$, where the height of each bar represents the average methylation levels of 10 or more CpGs, between 0 and $100 \%$ methylation.

The number of differentially methylated sites across all samples was smaller $(2,091)$ than the number of differentially methylated sites in the parents $(6,247$ and 8,480 in female and male parents, respectively), since the analysis across all samples was restricted to polymorphic reads, comprising, on average, $5 \%$ of all reads in our data. However, we observed that polymorphic fragments were much more likely to be differentially methylated than non-polymorphic fragments, since the fraction of differentially methylated CGs was 10 -fold higher in polymorphic sites (5.3\%) than in all CG sites in the parents $(0.56 \%$ in female and $0.52 \%$ in male parents). We also observed that the number of allele-specific methylation sites across all mice were highly correlated with SNP density (Pearson's $r=0.59$ ). To further explore this correlation, we compared the distribution of differentially methylated cytosines in polymorphic versus non-polymorphic regions. We counted the number of differentially methylated cytosines in 100-kb bins across the genome, and used a KS test to compare the distribution of the counts in nonpolymorphic versus polymorphic bins, containing at least one SNP. The average number of differentially methylated cytosines across all genomic bins was 17 -fold higher (0.77/ 0.045 ) in polymorphic bins relative to non-polymorphic bins in female B6 versus DBA mice (KS test, $P<1 \times 10^{-16}$ ), and 10 -fold higher $\left(0.95 / 0.095\right.$, KS test, $\left.P<1 \times 10^{-16}\right)$ in male B6 versus DBA mice (Figure S2E,F in Additional file 2).

\section{Sex differences}

To identify sex-specific methylation sites, we examined how DNA methylation patterns differed between females and males. As discussed above, we used a $t$-test to compare average methylation levels of reads from each group, but in this sex-specific comparison, we compared groups of females and males independent of genotype. We selected a cutoff of $P<0.001$ and a difference in methylation between the sexes greater than $20 \%$ (FDR $<5 \%$ ), which showed a strong enrichment of sex-specific methylation sites in the $\mathrm{X}$ chromosome (56\% of sites; Figure $3 \mathrm{~A}$ ). Using these criteria, we found 1,113 cytosines (0.026\%) with sex-specific methylation, associated with a total of 396 genes. This corresponded to 1,082 CGs (0.14\%), 7 CHGs and $24 \mathrm{CHHs}$. As an example, we found several cytosines differentially methylated in the gene encoding LIM homeobox protein $9(\operatorname{Lh} \times 9)$ on chromosome 1 (Figure $3 \mathrm{~B})$. For 


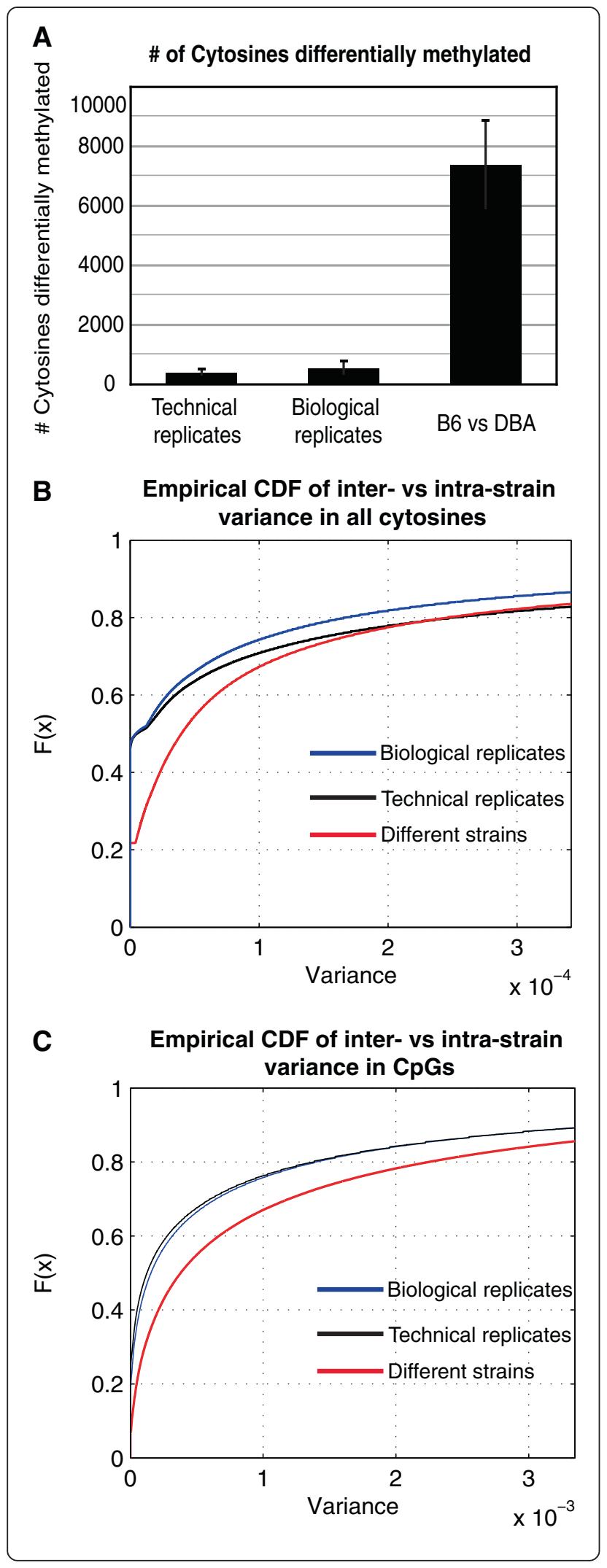

Figure $\mathbf{2}$ Reproducibility of methylation levels. (A) Differentially methylated cytosines identified in RRBS sequencing data derived from technical replicates, biological replicates, and samples from different mouse strains in B6 and DBA female or male mice. Error bars represent the standard deviation. $(\mathbf{B}, \mathbf{C})$ Distribution of the variance in methylation levels. The plots show the empirical cumulative distribution function (CDF) for the variance in methylation levels for all cytosines (B) and for CpGs (C). The distribution of biological replicates is shown by the blue curve, of technical replicates by the black curve, and of different mouse strains by the red curve.

each site, the height of each bar represents the average methylation levels of 10 or more CpGs, between 0 and $100 \%$ methylation. Notably, a previous study found that knock-out mice for this gene show infertility in both sexes, impaired gonad formation, and female-like genitalia in genetically male mice $[15,16]$. We examined cytosines differentially methylated between the sexes using the Genomic Regions Enrichment of Annotations Tool (GREAT), and found an enrichment in genes associated with regulation of histone methylation $\left(P=8.32 \times 10^{-11}\right)$, regulation of bone mineralization $\left(P=1.16 \times 10^{-8}\right)$, abnormal genitalia $\left(P=3.6870 \times 10^{-16}\right)$, decreased aggression towards other mice $\left(P=8.33 \times 10^{-4}\right)$ and X-linked inheritance $\left(P=1.56 \times 10^{-128}\right)$. A complete list of the 396 genes showing sex-specific methylation is provided in Additional file 4.

To determine whether sex-specific methylation coincides with expression differences, we examined liver expression levels in two published datasets, a BXD mouse cross [16], and a BXD F1 RNA-seq dataset from our laboratory [17]. We compared expression levels between females and males of the genes with sex-specific methylation. In the BXD cross, 237 genes out of the 396 differentially methylated genes were represented in the array, and 97 of these genes (41\% of all genes represented) were differentially expressed between females and males using a $t$-test $(P<0.05)$. Of these, 30 genes $(13 \%$ of all genes) were differentially expressed over 1.2-fold at FDR $<5 \%$. In the RNA-seq dataset, 45 out of the 396 differentially methylated genes were represented in the data. Of these, 13 genes (29\%) were differentially expressed between females and males over 1.2-fold $(P<0.05)$, and 5 of the genes $(11 \%)$ were differentially expressed at FDR $<5 \%$.

We carried out additional validation experiments to examine expression levels of nine genes differentially methylated between females and males. We measured expression levels using quantitative PCR (qPCR) on liver cDNA from 10 female and 6 male F1 mice, and compared expression levels between the two groups using a two-tailed $t$-test. For example, $C 4 a$ was differentially methylated, and we found that it was differentially expressed between females and males using both RNA-seq and qPCR (Figure 3C-E). Consistent with this finding, a 


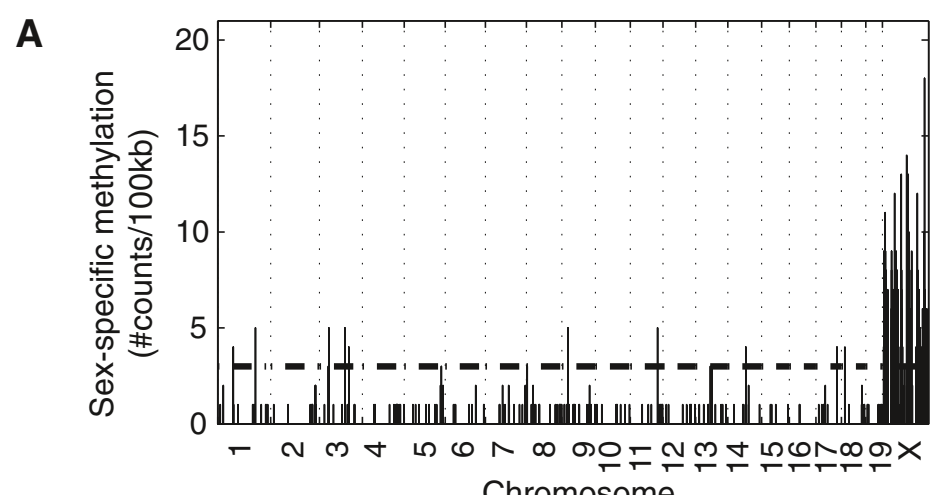

B
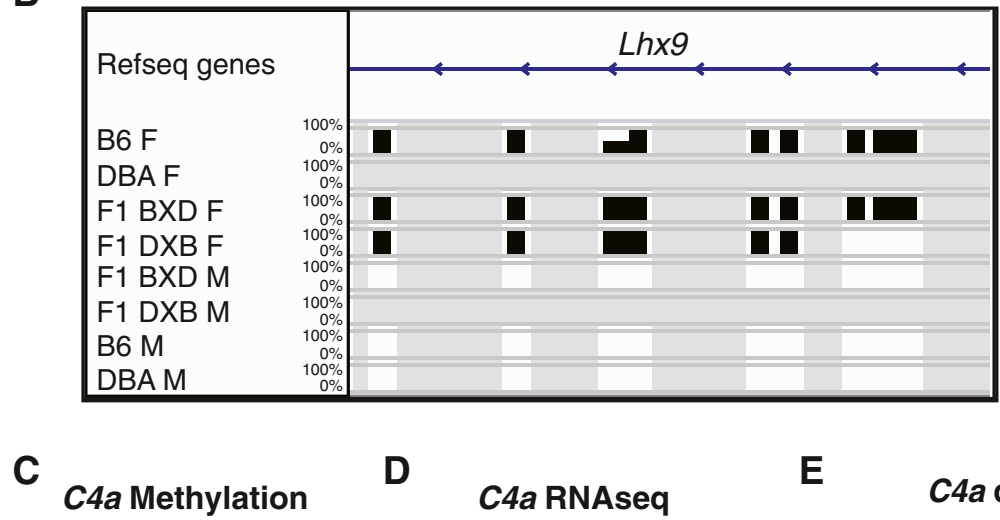

D
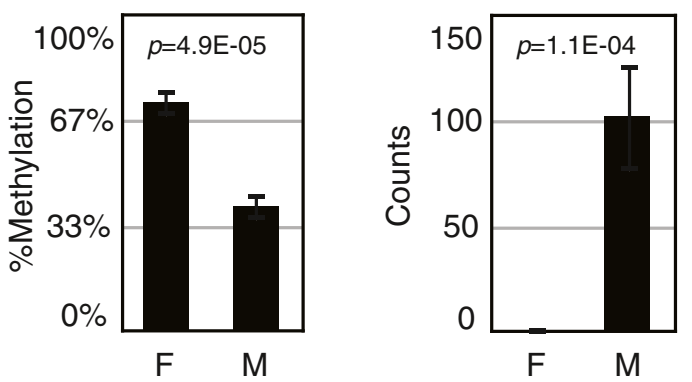

E

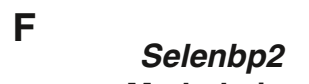

G Methylation

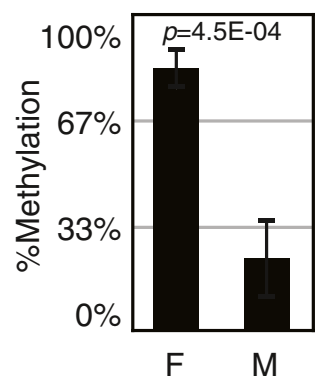

G Selenbp2 RNA

H Selenbp2 qPCR
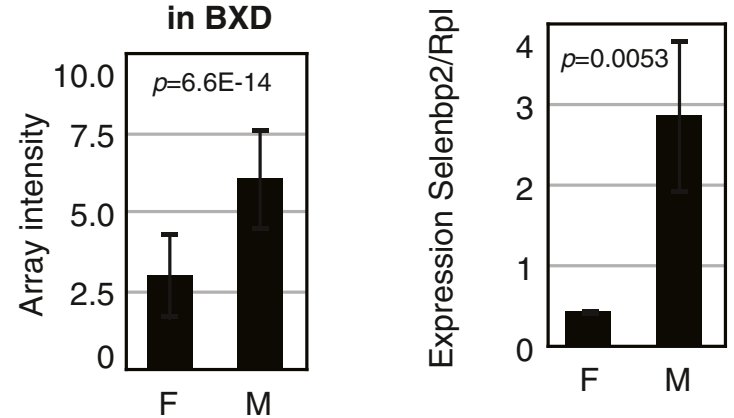

Figure 3 (See legend on next page.) 
(See figure on previous page.)

Figure 3 Sex differences. (A) Sex-specific methylation sites across the genome. The number of cytosines differentially methylated in each 100 -kb bin is shown on the $\mathrm{Y}$-axis and the genomic position of the bin is on the X-axis. All sites plotted are significant at 5\% FDR, and the horizontal dashed line represents the significance threshold for each bin. (B) Methylation levels across all samples for the gene $L h \times 9$. The height of the bar represents percentage methylation from 0 to 100\%. Gray represents missing data. (C) Average methylation levels in C4a at chr17:34960471. (D) Expression levels of C $4 a$ in RNA-seq dataset. (E) Expression levels of C4a measured by quantitative PCR. (F) Methylation levels of Selenbp2 at chr3:94499361. (G,H) Expression levels of Selenbp2 in BXD dataset $\mathbf{( G )}$ and by quantitative PCR $\mathbf{( H )}$. Error bars represent the standard deviation. F, females; $M$, males.

previous study found that an open chromatin structure in males correlated with increased C4a protein levels in the liver [18]. Also consistent with previous data [19], the gene Selenbp2, which encodes the major hepatic target for acetaminophen, Selenium-binding protein 2, was differentially methylated, and we also found that it was differentially expressed between males and females in the published BXD dataset and in our qPCR validation (Figure 3F-H). Overall, four out of nine genes measured by qPCR were differentially expressed between the two groups (Figure S4 in Additional file 5). Although the remaining genes were not statistically significant at $P<0.05$, they showed a trend towards differences between the two groups. It is possible that we did not have sufficient power to detect significant differences between the two groups using 16 F1 mice, as we did observe significant differences in the larger BXD expression array dataset. It is also possible that differences in methylation levels in some genes have functional consequences early in development but not in the adult animal, as suggested by large differences in methylation levels in $L x h 9$, but no significant differences in gene expression by qPCR in the adult liver. Thus, our findings suggest that DNA methylation is possibly a mechanism used to regulate sex-specific expression in a fraction of genes differentially methylated in the liver. A complete list of the genes differentially methylated and expressed is provided in Additional file 4.

\section{Imprinting}

DNA methylation is one of the mechanisms involved in genomic imprinting, whereby one of the two copies of a gene is expressed or silenced depending on the parent of origin. To identify imprinted genes, we first used the presence of polymorphic SNPs between the parental strains B6 and DBA in the sequencing reads to determine whether individual reads were derived from the father or the mother for each of the F1 mice. We then compared the average methylation level of maternal and paternal reads using a $t$-test and selected sites at the 5\% FDR cutoff. Using these criteria, we identified three previously known imprinted genes, H13, Impact and Snrpn, but no novel imprinted genes. As an example, methylation patterns in the $\mathrm{H} 13$ gene (Figure 4A) show that maternal chromosomes are highly methylated (B6 chromosomes in BXD mice and DBA chromosomes in DXB mice, shown in rows 1 to 4) while paternal chromosomes have low methylation levels (DBA chromosomes in BXD mice and B6 chromosomes in the DXB mice, shown in rows 5 to 8). The height of each bar represents the average methylation levels of 10 or more CpGs, between 0 and $100 \%$ methylation.

One likely reason that we were unable to find additional imprinted genes using this approach is that polymorphic reads represent only approximately 3 to $7 \%$ of the sequencing data we collected. To overcome this limitation, we used the method described by Fang et al. [20] to identify allelically methylated regions (AMRs), which is not limited to the use of polymorphic reads. The AMR approach uses a likelihood test to identify intervals of the genome that appear to have two alleles, each with a distinct methylation pattern. We selected AMRs where one allele was highly methylated and the other was not, where the difference in methylation between the two alleles was at least $50 \%$, and where the AMR was defined by at least 10 consecutive CpGs. Since the AMR approach is independent of genotype, it can identify both (a) allele-specific methylation that is dependent on the genotype of the DNA strand, and (b) imprinted regions that are dependent on the parental origin and independent of genotype. To focus on imprinted regions, we selected AMRs that were present in both the F1 mice and the parental mice, since the parental mice are inbred and both alleles have the same genotype on both alleles, and hence AMRs could not be caused by allelespecific methylation. Using this approach, we identified 123 putative imprinted protein-coding and microRNA genes, which included 11 known imprinted genes (enrichment $P=7.02 \times 10^{-11}$ ). This list provides a comprehensive set of putative imprinted loci, many of which have not been previously reported (Additional file 6).

To determine if these sites are associated with parentof-origin differential transcription, we tested for imprinting in the proximal genes using polymorphic reads from RNA-seq data in the F1 mice. Of the 123 genes, 32 were represented with 10 counts or more in the RNA-seq data. We tested for imprinting using Fisher's exact test and found $10(31 \%)$ of these had significantly different levels between the paternal and maternal copies $(P<0.05)$. As an example, we identified Met as a putative imprinted gene because there was an AMR in seven of the eight total 

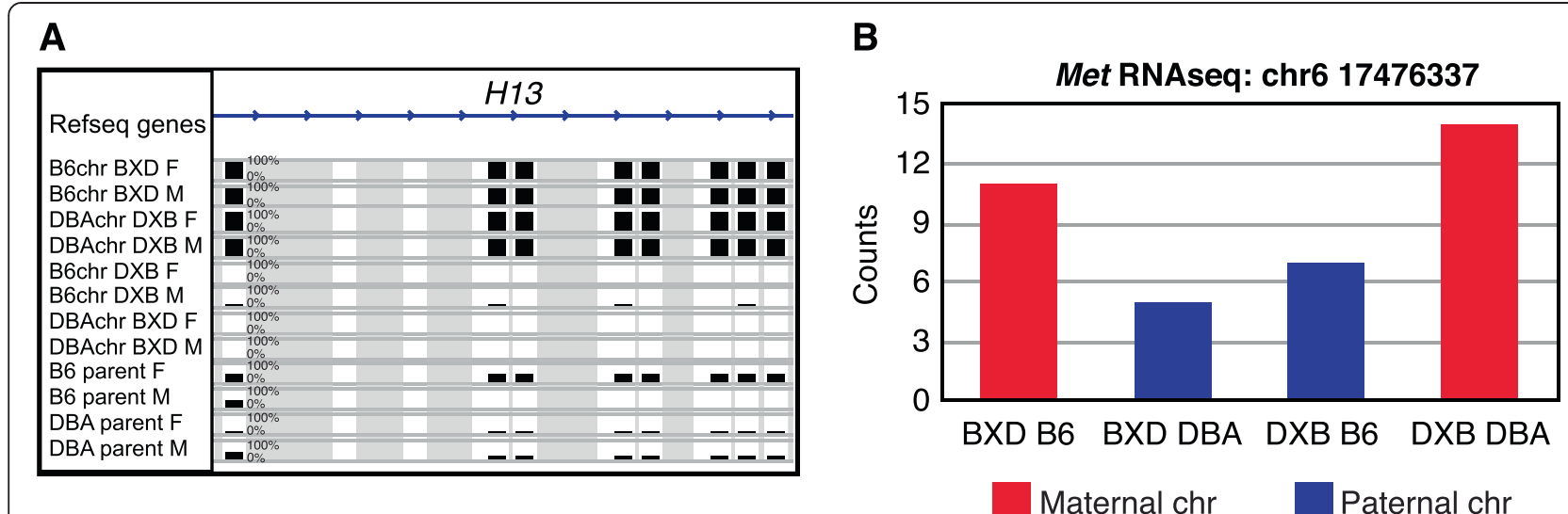

C Methylation in Met using RRBS

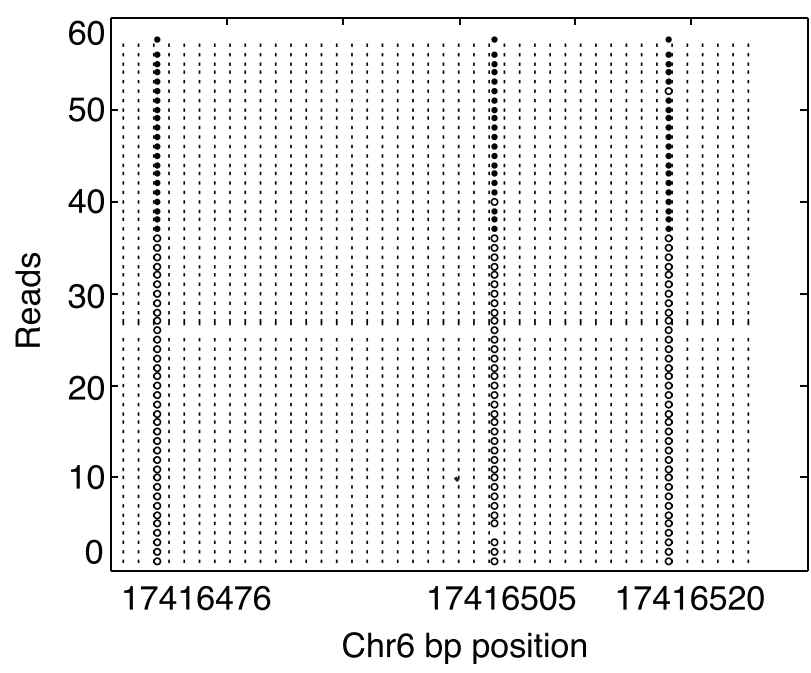

D

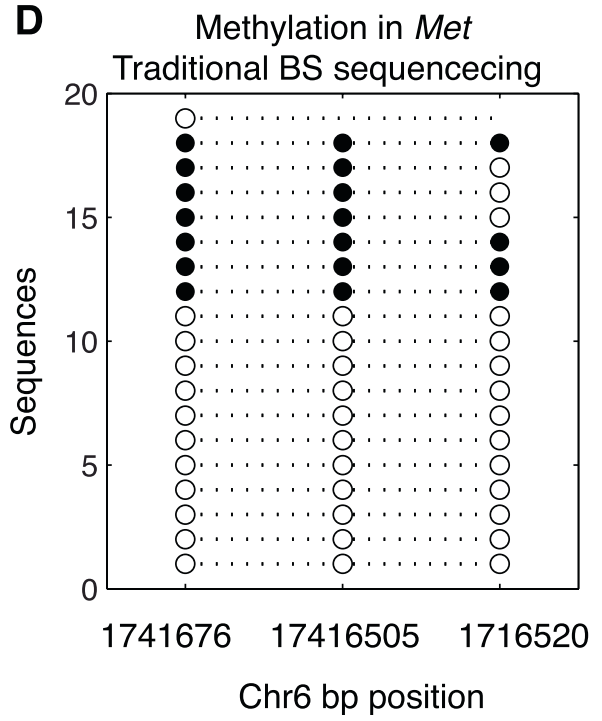

Figure 4 Imprinting. (A) Methylation levels across all samples, showing imprinting of the gene H13. The height of the bar represents percentage methylation from 0 to 100\%. Gray represents missing data. (B) Expression levels of Met measured by RNA-seq. (C) Methylation levels for an allelically methylated region (AMR) in the gene Met measured by RRBS sequencing. Each line represents CpGs in a sequencing read; open circles are unmethylated and filled circles are methylated CpGs. Reads are shown on the Y-axis and the genomic location of CpGs is shown on the X-axis. (D) Traditional bisulfite sequencing (BS) in the Met AMR. Sequences in different bacterial clones are on the $Y$-axis and the genomic location of $\mathrm{CpGs}$ is on the $\mathrm{X}$-axis.

parental and F1 samples (Figure 4C), and we confirmed Met imprinting using RNA-seq (Figure 4B). Furthermore, we carried out independent validation of our RRBS results using traditional bisulfite sequencing of the five putative novel imprinted genes Met, Nsd1, Vps37b, Oprd1 and Mapk15. We found that Met was differentially methylated using traditional bisulfite sequencing (Figure 4D), closely resembling the methylation patterns we observed in the RRBS data (Figure 4C). Similarly, the putative novel imprinted genes Nsd1, Vps37b, Oprd1 and Mapk15 were differentially methylated using traditional bisulfite sequencing (Additional file 7), consistent with our RRBS results.
A complete list of the known and novel imprinted genes we identified can be found in Additional file 6. Notably, we did not find imprinted genes or AMRs that were specific to either females or males. It is possible that we did not find evidence for imprinting in additional genes, or sex-specific differences due to lack of power. For instance, the known imprinted gene Copg2 was part of the list of 32 genes represented with 10 or more counts, but it did not pass the significance threshold. Furthermore, it is also possible that we did not find additional imprinted genes because of tissuespecific differences, such that additional imprinted genes may not necessarily be imprinted in the liver. 


\section{Intergenerational DNA methylation}

Intergenerational epigenetic marks are epigenetic marks, such as DNA methylation, that can be inherited from one organism to another. To examine the conservation and level of variation in intergenerational DNA methylation, we used a binomial test to compare methylation levels in B6 parents with those in B6 chromosomes in their F1 offspring, and in DBA parents with the DBA chromosomes in their F1 offspring. We found a total of $2,068(0.4 \%)$ intergenerational epimutations present in at least one parent-child pair that differed in methylation level by at least $50 \%$, and were below the $5 \%$ confidence interval of the binomial test. On average, we found 223 epimutations between parent-child pairs. When divided by category, we found, on average, 133 epimutations in female parent-child pairs and 303 in males, 173 in B6 and 232 in DBA chromosomes, with no significant differences between the groups (Figure S6A in Additional file 8). To determine if these epimutations were reproducible or stochastic, we selected sites that were reproducible in at least one or two parent-F1 comparisons (Figure S6C,D in Additional file 8). We found 973 (0.2\%) epimutations reproducible in at least one parent-F1 comparison, and $75(0.02 \%)$ epimutations in at least two parent-F1 comparisons, excluding sites associated with known imprinted genes. Notably, we found reproducible epimutations in at least two parent-F1 pairs only in B6 chromosomes and not in DBA chromosomes, which may reflect the fact that we have higher power to detect B6 epimutations as the reference genome we are using was derived from a B6 mouse.

The genomic distribution of epimutations can be found in Figure S6C,D in Additional file 8. Epimutations reproducible in at least one parent-F1 pair were generally distributed throughout the genome, although they tended to cluster in specific regions, or 'hotspots'. Epimutations reproducible in at least two parent-F1 pairs mostly clustered in two 'epimutation hotspots'. The first was located in the potassium channel-encoding gene Kcnip3 in chromosome 2 , a gene involved in neuronal excitability that was previously associated with Alzheimer's disease [21]. The second 'hotspot' was located the Obscurin gene (Obscn) in chromosome 11, a gene that may be involved in the formation of myofibrils. An example of a reproducible epimutation in the gene $W d r 63$ is shown in Figure S6B in Additional file 8). We conclude that, overall, reproducible intergenerational epigenetic changes in DNA methylation are rare, and that DNA methylation marks tend to be highly conserved between parents and offspring.

\section{Discussion}

In this study, we measured the extent of epigenetic variation among genetically distinct mice across two generations. We also surveyed expression data from similar samples to measure the degree to which these methylation differences are associated with expression changes. We found that DNA methylation was highly variable among genetically distinct individuals and differential methylation was strongly associated with polymorphic sites. We found thousands of cytosines differentially methylated between the parental mouse strains B6 and DBA (Figure 1B; Figure S2A in Additional file 2), and there was a strong enrichment for sites that are proximal to polymorphic alleles (Figure S2E,F in Additional file 2). Similarly, Xie et al. [4] found that $9.7 \%$ of CGs show allele-specific methylation between the more distantly related mouse strain $129 \mathrm{X} 1 / \mathrm{SvJ}$ and the mouse subspecies CAST/EiJ. This higher rate supports the notion that increased genetic variation is strongly associated with increased epigenetic variation [22].

Beyond the measurement of the association of genetic and epigenetic changes, our study design also allowed us to observe differences in DNA methylation between identical chromosomes across generations. Strikingly, we found that intergenerational epigenetic changes were exceedingly rare. With the exception of sites that were affected by imprinting, only 75 others were found to have reproducible differences between parents and F1 hybrid offspring in at least two parent- F1 comparisons. Thus, our overall results support the notion that (a) DNA methylation is highly heritable in mice, (b) there are minimal trans effects in F1 hybrids compared to their inbred parents, and (c) the majority of reproducible DNA methylation differences that accumulate between genetically identical mice are due to parent-of-origin effects. This result contrasts with the occurrence rate in plants, where intergenerational epigenetic changes appear to be more common, and are associated with RNA interference pathways [14].

Previous studies that examined sex-specific DNA methylation differences have typically focused on the X chromosome and have failed to identify significant differences in autosomes. Here, we compared DNA methylation patterns in females and males and found sex-specific methylation differences in 1,113 cytosines $(0.026 \%)$. We found that most of these (56\%) were on the X chromosome, but we also observed a large proportion of sex-specific methylation sites in autosomes. For example, we identified multiple sexspecific methylation changes in the autosomal gene $L h x 9$, and mouse knock-outs of this gene display defects in fertility and the development of genitalia and gonads. We also found differential methylation and expression of Maged1, and mouse knock-outs for this gene display impaired sexual behavior, decreased social interactions and hyperphagia [23]. A subset of the 396 differentially methylated genes also showed sex-specific expression differences in a previously published BXD mouse intercross and RNA-seq data from BXD/DXB F1 mice (Additional file 4). In summary, 
our results suggest that sex-specific epigenetic differences are found throughout the genome and can influence gene expression and physiological phenotypes. Since knock-outs for differentially methylated genes such as Lhx 9 and Maged 1 are impaired in sexual development and behavior, it is possible that specific epigenetic changes involved in sexual dimorphism are established early in development and reinforced by DNA methylation differences in adult animals.

Aberrant inheritance and expression of imprinted genes can result in clinical phenotypes such as Prader-Willi, Angelman and Turner syndromes. Currently, there are 150 known imprinted genes in mice, and we sought to identify novel imprinted genes. Differentially methylated regions (DMRs) controlling imprinted genes may be established early in development (gametic or primary DMRs) and are maintained in adult tissues, or established after fertilization (secondary or somatic DMRs) and may be tissue-specific [24]. Here we used a new approach to identify AMRs in regions where the genotype of the two chromosomes is not necessarily different [20]. The method relies on the identification of regions where the methylation of reads segregate into two populations, one highly methylated and one unmethylated. Using this approach we were able to identify a total of 123 imprinted genes. These included 11 known imprinted genes and 112 novel protein coding and microRNA genes, and we validated 10 putative novel imprinted genes using RNA-seq. Future studies are needed to examine the phenotypic consequences of aberrant imprinting in these novel regions.

\section{Conclusion}

Using RRBS sequencing in eight parental and F1 mice, we found novel imprinted genes, sex-specific DNA methylation in genes across the genome and allele-specific methylation. Our results strongly support the notion that, in mammals, the majority of variation in DNA methylation across individuals is associated with genetic differences, and is highly heritable from one generation to the next. Epigenetic factors have typically not been considered in genome-wide studies for complex traits, but our results suggest that DNA methylation is variable between individuals and is highly heritable, and hence can be a contributing factor to the heritability of traits. In the future it will be important to determine the contribution of epigenetic variation to the heritability of clinical traits, and the phenotypic impact of epigenetic variation on both molecular and complex clinical phenotypes.

\section{Materials and methods Mice}

Mice from strains C57BL/6J and DBA/2J were obtained from the Jackson Laboratories (Bar Harbor, ME, USA) and bred to generate F1 mice using reciprocal crosses, then housed in pathogen-free conditions and according to NIH guidelines. We collected livers from mice at 16 weeks of age, froze them in liquid nitrogen and stored them at $-80^{\circ} \mathrm{C}$. The mice were fasted overnight for 16 hours prior to euthanasia.

\section{Accession numbers}

RRBS sequencing data from this study, as well as all the results from strain-specific, allele-specific and sexspecific differentially methylated cytosines have been deposited in the NCBI GEO [25] under accession number [GEO:GSE53714].

\section{Reduced representation bisulfite sequencing libraries}

We used a previously described method for constructing RRBS libraries [26]. In brief, we isolated genomic DNA from livers using a phenol-chloroform extraction, digested genomic DNA using MspI restriction enzyme (NEB, Ipswich, MA, USA), then carried out end-repair/adenylation (NEB) and ligation with pre-methylated adapters (Illumina, San Diego, CA, USA). We selected fragments 250 to 350 bp in size using $2 \%$ agarose gel electrophoresis, purified the DNA from the gel fragments (QIAGEN, Valencia, CA, USA) and carried out bisulfite treatment on the DNA (Milipore, Billerica, MA, USA) followed by PCR amplification.

\section{Alignment}

We aligned reads using BS Seeker [12] to the $\mathrm{mm} 9$ mouse reference genome, keeping uniquely aligned reads.

\section{SNP calling}

We pooled data from B6, DBA and BXD F1 samples for A, C, G and T counts at each position observed. We called SNPs for loci with a minimum of six counts and a minor allele frequency of $10 \%$, excluding C/T SNPs, and cross-checked our calls with public sequencing data for these strains [13]. We observed a total of 24,132 SNPs in our data. For each sample, approximately 3 to $7 \%$ of the reads were polymorphic.

\section{Simulations}

We simulated methylation counts to estimate FDR in methylation levels. For a specific site, we simulated binomially distributed data using the total number of counts $(n)$ and the average methylation level $(p)$ at that site, using binornd with parameters $n$ and $p$ in MATLAB. We took the ratio between the number of sites that were significant by chance in the simulation data $(x)$ over the number of significant sites in the real data $(y)$ as the simulation-based FDR $(x / y)$.

\section{Binomial test}

We selected data from cytosines covered by 10 or more reads. To test for differences in methylation at specific 
sites between individual samples, we used the binomial distribution with parameters $p=$ percent methylation at that site, and $n=$ total number of counts, and estimated 95\% confidence intervals using binofit in MATLAB [14]. Sites were considered differentially methylated between two samples if the mean methylation of each sample was outside of the $95 \%$ confidence interval of the other sample, and if the difference between the mean methylation delta was $>50 \%$. This corresponded to a simulationbased FDR $<1 \%$.

\section{Inter-strain versus intra-strain variance}

To compare the distribution of the variance in methylation levels, we first computed the variance in methylation levels for each cytosine across RRBS libraries of difference strains (inter-strain variance), the variance in methylation levels between samples derived from different mice of the same strain (intra-strain variance for biological replicates), or the variance in methylation levels between different libraries made from the same mouse genomic DNA sample (technical replicates). We then compared the distribution of these variances using a KS test (kstest2 function in MATLAB), and visualized the distributions using the cumulative distribution function (cdfplot function in MATLAB). We calculated the mean of the variance in methylation across all cytosines to compare the fold difference between the distributions, and determined that the mean of the inter-strain variance was nine-fold higher than the intra-strain variance in biological replicates. We also selected data only from CG cytosines to compute the mean of the variance in methylation levels, and found that the mean variance in CG methylation was 2.4-fold higher between strains than between biological replicates.

\section{Allele-specific methylation}

We tested for allele-specific methylation between groups of B6 and DBA samples using a $t$-test. We used our called SNPs to determine if reads were derived from $B 6$ or DBA chromosomes, and determined the percentage methylation levels for each. We compared percentage methylation levels between B6 and DBA chromosomes using a $t$-test. Sites were considered differentially methylated if $P<0.05$ and delta between B6 and DBA groups was $>50 \%$. This corresponded to FDR $<0.05 \%$ based on 1,000 simulations.

\section{Sex differences}

We tested for sex differences using a $t$-test as described above, but we tested for differences in sites using reads derived from females versus males. Sites were considered differentially methylated if $P<0.001$ and delta between females and males was $>20 \%$, which corresponded to FDR $<5 \%$. We used the $\mathrm{mm} 9$ genome annotation [27] to identify 396 genes located within $10 \mathrm{~kb}$ of differentially methylated sites.

\section{Quantitative PCR (qPCR)}

We purified RNA from livers of 10 female and 6 male F1 mice using Trizol (Life Technologies, Grand Island, NY, USA) and synthesized cDNA using $1 \mu \mathrm{g}$ of RNA and a cDNA synthesis kit (Life Technologies), then diluted the cDNA 20 times and used $3 \mu \mathrm{l}$ of the diluted cDNA per qPCR reaction. For each gene target, we measured each sample in triplicate using the KAPA Biosystems SYBR Green Mix (Wilmington, MA, USA) in a $12 \mu \mathrm{l}$ reaction volume and the following conditions: $95^{\circ} \mathrm{C}$ for 5 minutes, and 50 cycles of $95^{\circ} \mathrm{C}$ for 15 seconds, $60^{\circ} \mathrm{C}$ for 15 seconds, and $72^{\circ} \mathrm{C}$ for 15 seconds. We determined the absolute quantity of each transcript using a standard curve in the Roche Light Cycler 480 program, and normalized the quantity of each sample relative to the quantity of the house-keeping gene $R p l$. We compared average levels of each transcript between females and males using a two-tailed $t$-test.

\section{Imprinting}

We used our called SNPs to determine whether reads in F1 mice were derived from B6 or DBA chromosomes, and classified reads as derived from maternal or paternal for both reciprocal F1 types BXD and DXB. We compared percentage methylation levels at individual sites in maternal versus paternal chromosomes using a $t$-test as described above. Sites were considered imprinted if $P<$ $1 \times 10^{-6}$, which corresponded to FDR $<50 \%$ based on 1,000 simulations, but resulted in only three known imprinted genes.

\section{Allelically methylated regions}

AMRs were identified by the computational method described by Fang et al. [20] with the tool 'amrfinder'. The tool uses a fixed-width window (that is, fixed number of CpG sites) to identify AMRs genome-wide. For each window, the likelihood values of observed reads from bisulfite sequencing are calculated by assuming the region is or is not an AMR. Then the likelihood ratio test is employed to determine the more appropriate model for the region. The underlying probabilistic model describes the degree to which reads appear to have two distinct methylation patterns and is independent of genotype. We selected AMRs called using a window size of 10 consecutive CpGs, $P<0.01$ and delta between the two methylation patterns was greater than $50 \%$, and considered the AMR as a putative imprinted region if it was present in at least 6 of the 8 total samples, including parents and F1 mice. Enrichment in imprinted genes was calculated using the hypergeometric test, $p=1$-hygecdf in MATLAB. 


\section{RNA-seq}

\section{Imprinting}

RNA-seq data from the livers of 11 mice were recently published by Lagarrigue et al. [17], and included 6 BXD, 5 DXB, 6 females and 5 males. The authors used SNPs in polymorphic reads to determine parental origin of the read, and we used these RNA-seq counts to look for evidence for imprinting using a Fisher's exact test, where the variables are cross direction (BXD, DXB) and genotype (B6, DBA), and selected specific sites where FDR $<5 \%$ using the false discovery approach introduced by Storey [28], and where the fold difference between the two sexes was greater than 1.2-fold.

\section{Sex differences}

To test for sex differences in the RNA-seq dataset, we used a $t$-test to compare counts at specific sites between female and male F1 mice, and estimated FDR using the Storey method [28].

\section{Expression in BXD intercross}

We obtained Agilent array expression data from a published BXD intercross composed of a total of 122 mice, parental, F1 and F2 mice, 72 females and 50 males [16]. We used a $t$-test to compare females versus males for individual genes, and estimated FDR using the Storey method [28]. We selected genes at a FDR $<5 \%$ cutoff, and where the fold difference between females and males was greater than 1.2-fold.

\section{GREAT}

We looked for enriched biological processes and phenotype annotations using GREAT [29]. To annotate fragments with sex-specific methylation, we used the list of DNA fragments that were differentially methylated between females and males as our test list, and all the fragments represented in our RRBS data as the reference list.

\section{Traditional bisulfite sequencing}

We carried out bisulfite treatment using $1 \mu \mathrm{g}$ of liver genomic DNA from BXD or DXB F1 male mice. We designed degenerate primers (IDT) to amplify bisulfitetreated DNA with the Bisulfite Primer Seeker program from Zymo (Irvine, CA, USA). We amplified each target gene with MyTaq (Bioline), using $2 \mu \mathrm{L}$ of DNA in 50 PCR reaction volume, and the following PCR conditions: $95^{\circ} \mathrm{C}$ for 2 min; 2 cycles of $95^{\circ} \mathrm{C}$ for $30 \mathrm{~s}, 62^{\circ} \mathrm{C}$ for $90 \mathrm{~s}$ and $62^{\circ} \mathrm{C}$ for $2 \mathrm{~min} ; 2$ cycles of $95^{\circ} \mathrm{C}$ for $30 \mathrm{~s}, 61^{\circ} \mathrm{C}$ for $90 \mathrm{~s}$ and $61^{\circ} \mathrm{C}$ for 2 min; 2 cycles of $95^{\circ} \mathrm{C}$ for $30 \mathrm{~s}, 60^{\circ} \mathrm{C}$ for $90 \mathrm{~s}$ and $60^{\circ} \mathrm{C}$ for $2 \mathrm{~min}$; 2 cycles of $95^{\circ} \mathrm{C}$ for $30 \mathrm{~s}$, $59^{\circ} \mathrm{C}$ for $90 \mathrm{~s}$ and $60^{\circ} \mathrm{C}$ for $2 \mathrm{~min}$; 31 cycles of $95^{\circ} \mathrm{C}$ for $30 \mathrm{~s}, 58^{\circ} \mathrm{C}$ for $90 \mathrm{~s}$ and $60^{\circ} \mathrm{C}$ for $2 \mathrm{~min}$; final extension at $60^{\circ} \mathrm{C}$ for $15 \mathrm{~min}$. We cloned PCR products from each sample using the TOPO cloning kit for sequencing
(Life Technologies, Grand Island NY, USA) according to the manufacturer's instructions. We plated transformed bacterial on Luria broth plates containing $50 \mu \mathrm{g} / \mathrm{ml}$ kanamycin and $20 \mu \mathrm{g} / \mathrm{ml} \mathrm{X-gal} \mathrm{overnight} \mathrm{at}$ $37^{\circ} \mathrm{C}$ in the dark. We picked $15-20$ white colonies from each transformation and submitted the clones for Sanger sequencing to Genewiz (La Jolla, CA, USA). We aligned the sequences using multiple alignment in the CLC Main Workbench program.

\section{Additional files}

Additional file 1: Figure S1. RRBS data quality. (A) Total number of reads and uniquely aligned reads in each RRBS library. (B) Mappability of each library, or fraction of uniquely aligned reads by BS-Seeker. (C) Average coverage in cytosines. (D) Sequencing error rate in reads as the percentage of reads that do not match expected genotypes in libraries from B6 and DBA mice (Reads with error). (E,F) Genome-wide average methylation levels for each sample in (E) CG, (F) CHG and $\mathrm{CHH}$ contexts.

Additional file 2: Figure S2. Strain specific differences. (A) Differentially methylated cytosines in B6 and DBA male parents. The number of cytosines differentially methylated in each 100-kb bin is shown on the Y-axis and the genomic position of the bin is on the $X$-axis. All sites plotted are significant at $1 \% \mathrm{FDR}$, and the horizontal dashed line represents the significance threshold for each bin. $(\mathbf{B}, \mathbf{C})$ Fraction of differentially methylated cytosines in each context in (B) female and (C) male B6 and DBA strains. (D) Overlap of differentially methylated cytosines identified in B6 and DBA female mice, or B6 and DBA male mice. (E,F) The distribution of the number of differentially methylated cytosines in (E) B6 and DBA females and (F) B6 and DBA males. The cumulative distribution function for the number of cytosines differentially methylated is shown for non-polymorphic bins, and polymorphic bins containing at least one SNP.

Additional file 3: Figure S3. Cytosine methylation clusters by genotype of chromosomes. Hierarchical clustering of samples based on methylation levels from 38,427 cytosine sites. Individual B6 or DBA chromosomes in BXD mice were determined based on the genotype of polymorphic SNPS present in the read.

Additional file 4: Table S1. Genes differentially methylated by sex.

Additional file 5: Figure S4. Validation of sexual dimorphism by $q P C R$. (A-I) Expression levels measured by qPCR on mouse liver cDNA for nine genes differentially methylated between females and males. Expression levels of each gene are plotted on the $Y$-axis relative to the house-keeping gene Rpl. Each bar represents the average of 10 female (F) and 6 male (M) mice.

Additional file 6: Table S2. Imprinted genes identified through AMRs.

Additional file 7: Figure S5. Validation of AMRs by traditional bisulfite sequencing. (A-D) Traditional bisulfite sequencing for AMRs in (A) Nsd1, (B) Vps376, (C) Oprd1 and (D) Mapk15. Sequences in different bacterial clones are on the $Y$-axis and the genomic location of CpGs is on the X-axis. Open circles are unmethylated and filled circles are methylated CpGs.

Additional file 8: Figure S6. Intergenerational methylation differences. (A) Average number of epimutations between parent and F1s. The average of parent-F1 comparisons is shown for all, or specifically for comparisons between females, males, B6 or DBA chromosomes. (B) Methylation levels across all samples in the gene Wdr63, showing variation in B6 chromosomes from different samples. BXD are F1 mice of B6 female and DBA male parents, $D X B$ are $F 1$ mice of DBA female of $B 6$ male parents. The height of the bar represents percentage methylation from 0 to 100\%. Gray represents missing data. (C,D) Reproducible epimutations across the genome identified in at least one (C) or two (D) parent- F1 comparisons. The number of epimutations in each 100-kb bin is shown on the $\mathrm{Y}$-axis and the genomic position of the bin is on the $X$-axis. The horizontal dashed line represents the significance threshold for each bin. 


\section{Abbreviations}

AMR: allelically methylated region; B6: C57BL/6J mouse strain; BXD: C57BL/6J x DBA/2J F1 mouse, where C57BL/6J was the female parent and DBA/2J was the male parent; DBA: DBA/2J mouse strain; DMR: differentially methylated region; DXB: DBA/2J x C57BL/6J F1 mouse, where DBA/2J was the female parent and $\mathrm{C} 57 \mathrm{BL} / 6 \mathrm{~J}$ was the male parent; FDR: false discovery rate; KS: Kolmogorov-Smirnov; PCR: polymerase chain reaction; qPCR: quantitative PCR; RRBS: reduced representation bisulfite sequencing; SNP: single nucleotide polymorphism

\section{Competing interests}

The authors declare that they have no competing interests.

\section{Authors' contributions}

LDO performed the analyses and wrote the paper. LR constructed the sequencing libraries. LM bred the mice and collected the tissues. FF and ADS performed the analyses on the AMRs. FH aligned the RNA-seq data. LDO and NC performed traditional bisulfite sequencing experiments. AJL, MP and LDO conceived the project, and helped craft and edit the manuscript. MP directed the project. All authors read and approved the final manuscript.

\section{Acknowledgements}

This work was supported by the National Institutes of Health (grant number GM095656-01A1).

\section{Author details}

'Department of Molecular, Cell and Developmental Biology, University of California Los Angeles, Los Angeles, CA 90095, USA. ²Department of Medicine, University of California Los Angeles, Los Angeles, CA 90095, USA. ${ }^{3}$ Molecular and Computational Biology, University of Southern California, Los Angeles, CA 90089, USA. ${ }^{4}$ Department of Computer Science, University of California Los Angeles, Los Angeles, CA 90095, USA

Received: 8 August 2013 Accepted: 30 April 2014 Published: 30 April 2014

\section{References}

1. Hannum G, Guinney J, Zhao L, Zhang L, Hughes G, Sadda S, Klotzle B, Bibikova M, Fan JB, Gao Y, Deconde R, Chen M, Rajapakse I, Friend S, Ideker T, Zhang K: Genome-wide methylation profiles reveal quantitative views of human aging rates. Mol Cell 2013, 49:359-367.

2. Liu Y, Aryee MJ, Padyukov L, Fallin MD, Hesselberg E, Runarsson A, Reinius L, Acevedo N, Taub M, Ronninger M, Shchetynsky K, Scheynius A, Kere J, Alfredsson L, Klareskog L, Ekström TJ, Feinber AP: Epigenome-wide association data implicate DNA methylation as an intermediary of genetic risk in rheumatoid arthritis. Nat Biotechnol 2013, 31:142-147.

3. Lister R, Pelizzola M, Dowen RH, Hawkins RD, Hon G, Tonti-Filippini J, Nery JR, Lee L, Ye Z, Ngo QM, Edsall L, Antosiewicz-Bourget J, Stewart R, Ruotti V, Millar AH, Thomson JA, Reng B, Ecker JR: Human DNA methylomes at base resolution show widespread epigenomic differences. Nature 2009, 462:315-322.

4. Xie W, Barr CL, Kim A, Yue F, Lee AY, Eubanks J, Dempster EL, Ren B: Base-resolution analyses of sequence and parent-of-origin dependent DNA methylation in the mouse genome. Cell 2012, 148:816-831.

5. Feng S, Cokus SJ, Zhang X, Chen PY, Bostick M, Goll MG, Hetzel J, Jain J, Strauss SH, Halpern ME, Ukomadu C, Sadler KC, Pradhan S, Pellegrini M, Jacobsen SE: Conservation and divergence of methylation patterning in plants and animals. Proc Natl Acad Sci USA 2010, 107:8689-8694.

6. Lister R, Pelizzola M, Kida YS, Hawkins RD, Nery JR, Hon G, Antosiewicz-Bourget J, O'Malley R, Castanon R, Klugman S, Downes M, Yu R, Stewart R, Ren B, Thomson JA, Evans RM, Ecker JR: Hotspots of aberrant epigenomic reprogramming in human induced pluripotent stem cells. Nature 2011, 471:68-73.

7. Deaton $\mathrm{AM}, \mathrm{Bird} \mathrm{A}: \mathrm{CpG}$ islands and the regulation of transcription. Genes Dev 2011, 25:1010-1022.

8. Silva AJ, White R: Inheritance of allelic blueprints for methylation patterns. Cell 1988, 54:145-152

9. Lane N, Dean W, Erhardt S, Hajkova P, Surani A, Walter J, Reik W: Resistance of IAPs to methylation reprogramming may provide a mechanism for epigenetic inheritance in the mouse. Genesis 2003, 35:88-93.
10. Morgan $\mathrm{HD}$, Sutherland $\mathrm{HG}$, Martin DI, Whitelaw E: Epigenetic inheritance at the agouti locus in the mouse. Nat Genet 1999, 23:314-318.

11. Schilling E, El Chartouni C, Rehli M: Allele-specific DNA methylation in mouse strains is mainly determined by cis-acting sequences. Genome Res 2009, 19:2028-2035.

12. Chen PY, Cokus SJ, Pellegrini M: BS Seeker: precise mapping for bisulfite sequencing. BMC Bioinforma 2010, 11:203.

13. Keane TM, Goodstadt L, Danecek P, White MA, Wong K, Yalcin B, Heger A, Agam A, Slater G, Goodson M, Furlotte NA, Eskin E, Nellåker C, Whitley H, Cleak J, Janowitz D, Hernandez-Pliego P, Edwards A, Belgard TG, Oliver PL, McIntyre RE, Bhomra A, Nicod J, Gan X, Yuan W, van der Weyden L, Steward CA, Bala S, Stalker J, Mott R, et al: Mouse genomic variation and its effect on phenotypes and gene regulation. Nature 2011, 477:289. 294.

14. Chodavarapu RK, Feng S, Ding B, Simon SA, Lopez D, Jia Y, Wang GL, Meyers BC, Jacobsen SE, Pellegrini M: Transcriptome and methylome interactions in rice hybrids. Proc Natl Acad Sci USA 2012, 109:12040-12045.

15. Birk OS, Casiano DE, Wassif CA, Cogliati T, Zhao L, Zhao Y, Grinberg A, Huang S, Kreidberg JA, Parker KL, Porter FD, Westphal H: The LIM homeobox gene Lhx9 is essential for mouse gonad formation. Nature 2000, 403:909-913.

16. Gatti DM, Zhao N, Chesler EJ, Bradford BU, Shabalin AA, Yordanova R, Lu L, Rusyn I: Sex-specific gene expression in the BXD mouse liver. Physiol Genomics 2010, 42:456-468.

17. Lagarrigue S, Hormozdiari F, Martin L, Lecerf F, Hasin Y, Rau C, Hagopian R, Xiao Y, Yan J, Drake TA, Ghazalpour A, Eskin E, Lusis AJ: Limited RNA editing in exons of mouse liver and adipose. Genetics 2013, 193:1107-1115.

18. Hemenway C, Robins DM: DNase I-hypersensitive sites associated with expression and hormonal regulation of mouse $\mathrm{C} 4$ and Slp genes. Proc Natl Acad Sci USA 1987, 84:4816-4820.

19. Mattow J, Demuth I, Haeselbarth G, Jungblut PR, Klose J: Selenium-binding protein 2, the major hepatic target for acetaminophen, shows sex differences in protein abundance. Electrophoresis 2006, 27:1683-1691.

20. Fang F, Hodges E, Molaro A, Dean M, Hannon GJ, Smith AD: Genomic landscape of human allele-specific DNA methylation. Proc Natl Acad Sci USA 2012, 109:7332-7337.

21. Jo DG, Lee JY, Hong YM, Song S, Mook-Jung I, Koh JY, Jung YK: Induction of pro-apoptotic calsenilin/DREAM/KChIP3 in Alzheimer's disease and cultured neurons after amyloid-beta exposure. J Neurochem 2004, 88:604-611.

22. Bell JT, Pai AA, Pickrell JK, Gaffney DJ, Pique-Regi R, Degner JF, Gilad Y, Pritchard JK: DNA methylation patterns associate with genetic and gene expression variation in HapMap cell lines. Genome Biol 2011, 12:R10

23. Dombret C, Nguyen T, Schakman O, Michaud JL, Hardin-Pouzet H, Bertrand MJ, De Backer O: Loss of Maged1 results in obesity, deficits of social interactions, impaired sexual behavior and severe alteration of mature oxytocin production in the hypothalamus. Hum Mol Genet 2012, 21:4703-4717.

24. Henckel A, Arnaud P: Genome-wide identification of new imprinted genes. Brief Funct Genomics 2010, 9:304-314.

25. Edgar R, Domrachev M, Lash AE: Gene Expression Omnibus: NCBI gene expression and hybridization array data repository. Nucleic Acids Res 2002, 30:207-210

26. Feng S, Rubbi L, Jacobsen SE, Pellegrini M: Determining DNA methylation profiles using sequencing. Methods Mol Biol 2011, 733:223-238.

27. Kent WJ, Sugnet CW, Furey TS, Roskin KM, Pringle TH, Zahler AM, Haussler D: The human genome browser at UCSC. Genome Res 2002, 12:996-1006.

28. Storey JD: A direct approach to false discovery rates. J Roy Stat Soc B 2002, 64:479-498.

29. McLean CY, Bristor D, Hiller M, Clarke SL, Schaar BT, Lowe CB, Wenger AM, Bejerano G: GREAT improves functional interpretation of cis-regulatory regions. Nat Biotechnol 2010, 28:495-501.

doi:10.1186/gb-2014-15-5-r68

Cite this article as: Orozco et al:: Intergenerational genomic DNA methylation patterns in mouse hybrid strains. Genome Biology 2014 15:R68 